\title{
DETERMINANTES DE LA DESERCIÓN ESTUDIANTIL EN ESTUDIANTES UNIVERSITARIOS
}

Miguel Camacho Manjarrez

Universidad de Cartagena

Colombia

Amparo Montalvo Prieto Universidad de Cartagena Colombia

Patricia Galezo Arango Universidad de Cartagena Colombia 
Panorama Económico, Vol. 27 - No. 1 (Enero - Marzo de 2019), pp. 134-162

Miguel Camacho Manjarrez

Amparo Montalvo Prieto

Patricia Galezo Arango

JEL: I21, I23, I29

\title{
Determinantes de la deserción estudiantil en estudiantes universitarios
}

\section{Resumen}

La deserción estudiantil es considerada un problema para el Sistema Educativo. El presente estudio tuvo como objetivo analizar la deserción estudiantil y la incidencia de los factores determinantes en estudiantes de pregrado de la Universidad de Cartagena. Es un estudio descriptivo, correlacional que caracteriza a los 16.927 estudiantes de pregrado matriculados en la Universidad de Cartagena en diversas cohortes. Se establecieron los posibles determinantes en el riesgo de deserción y patrones temporales de deserción. Los datos se consultaron en la plataforma de SPADIES, previa autorización de la Oficina de Planeación. La muestra fue de 1547 estudiantes desertores de la Universidad de Cartagena y fue proporcional a cada programa y se utilizó un instrumento de 22 preguntas, elaborado por los investigadores. La tasa de deserción en la Universidad de Cartagena ha disminuido y se encuentra en correspondencia con el comportamiento de la tasa promedio de deserción a nivel nacional. Presentan más riesgo de desertar los hombres y los estudiantes de los programas de la modalidad a distancia, quienes ingresan con una clasificación baja en las pruebas Saber 11 y presentan un mayor riesgo en los primeros períodos de estudio.

Palabras clave: Estudiante, educación superior, deserción, ayuda educativa

\section{Déterminants du décollement des étudiants chez les étudiants de premier cycle}

\section{Résumé}

La désertion des élèves est considérée comme un problème pour le système éducatif. L'objectif de cette étude était d'analyser la désertion des étudiants et l'incidence des facteurs déterminants chez les étudiants de premier cycle à l'Université de Carthagène. Il s'agit d'une étude descriptive et corrélationnelle qui caractérise les 16927 étudiants de premier cycle inscrits à l'Université de Carthagène dans diverses cohortes. Les déterminants possibles du risque de décrochage et des modèles de décrochage temporel ont été établis. Les données ont été consultées sur la plateforme SPADIES, avec l'autorisation préalable du Bureau de l'urbanisme. L'échantillon était composé de 1547 étudiants décrocheurs de l'Université de Cartagena et était proportionnel à chaque programme et un instrument de 22 questions a été utilisé, préparé par les chercheurs. Le taux d'abandon à l'Université de Carthagène a diminué et correspond au comportement du taux d'abandon moyen à l'échelle nationale. Les hommes et les étudiants des programmes d'enseignement à distance sont plus à risque d'abandonner, qui entrent avec une faible classification dans les tests Sabre 11 et ont un risque plus élevé dans les premières périodes d'étude.

Mots clés: Étudiant, enseignement supérieur, abandon, aide à l'éducation

\section{Determinants of student dropout in undergraduate students}

\begin{abstract}
Student desertion is considered a problem for the Educational System. The objective of this study was to analyze student desertion and the incidence of determining factors in undergraduate students at the University of Cartagena. It is a descriptive, correlational study that characterizes the 16,927 undergraduate students enrolled at the University of Cartagena in various cohorts. Possible determinants of dropout risk and temporal dropout patterns were established. The data was consulted on the SPADIES platform, with prior authorization from the Planning Office. The sample was 1547 dropout students from the University of Cartagena and was proportional to each program and an instrument of 22 questions was used, prepared by the researchers. The dropout rate at the University of Cartagena has decreased and is in correspondence with the behavior of the average dropout rate nationwide. Men and students from distance learning programs are more at risk of dropping out, who enter with a low classification in the Saber 11 tests and have a higher risk in the first study periods.
\end{abstract}

Keywords: Student, higher education, dropout, educational aid 


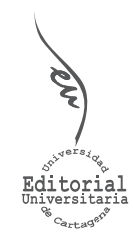

Artículo de Investigación

\section{Determinantes de la deserción estudiantil en estudiantes universitarios}

\section{INFORMACIÓN DEL ARTíCULO}

Recepción del artículo: 06/07/2018

Concepto de evaluación: 30/08/2018

Aceptación del artículo: 27/09/2018
Miguel Camacho Manjarrez Universidad de Cartagena, Colombia

Amparo Montalvo Prieto* Universidad de Cartagena, Colombia

Patricia Galezo Arango Universidad de Cartagena, Colombia

\section{INTRODUCCIÓN}

La deserción estudiantil es considerada como el abandono definitivo de la formación académica por parte de los estudiantes, independientemente de las condiciones y la modalidad (Paramo, 1999). Este fenómeno es considerado un problema para el Sistema Educativo, lo que hace obligatorio que en las instituciones se establezcan mecanismos de ajuste a la vida universitaria de sus estudiantes, impactando así en el éxito académico.

Por tal razón, es importante desarrollar estudios que permitan determinar la dimensión del problema en la institución educativa y de acuerdo a ello poder establecer con la debida anticipación, estrategias orientadas a lograr la finalización del programa que se está cursando. La graduación estudiantil es el reflejo del cumplimiento de los objetivos fundamentales de la Educación Superior y se logra a partir del desarrollo de procesos formativos y la implementación de estrategias de intervención pertinentes en cada etapa del ciclo de formación del estudiante (Ministerio de Educación Nacional, 2015).

En Colombia de acuerdo a información del Ministerio de Educación Nacional (MEN), se han alcanzado avances en cobertura y acceso al sistema de educación superior, pero preocupa el número de estudiantes que ingresan y no culminan sus estudios superiores (Ministerio de Educación Nacional, 2015). En el 2013 la tasa de deserción en programas universitarios fue del 44.9\%, mientras que en los programas técnicos y tecnológicos llegó al 62.4\% y 53.8\%. La Organización para la Cooperación y el Desarrollo Económicos (OCDE), considera que la deserción es un problema de eficiencia y de equidad del sistema educativo, por el malgasto del dinero invertido y el no cumplimiento de las necesidades de mano de obra calificada (Ministerio de Educación Nacional, 2015).

\footnotetext{
*Autor de correspondencia: amontalvop1@unicartagena.edu.co
} 
El Ministerio de Educación Nacional, realiza el seguimiento a la deserción a través de dos indicadores, la tasa de deserción anual y por cohorte. En la tasa de deserción anual Colombia registra una tasa de $10.4 \%$, superior a la del Reino Unido $8.6 \%$ e inferior a la de Brasil 18\% y Estados Unidos 18.3\%, con relación a la tasa de deserción por cohorte, que muestra la no culminación de estudios en un programa determinado, Colombia alcanza una cifra de $44.9 \%$, superior a la tasa de México 42\% y Argentina 43\% y muy por debajo de las tasas de Venezuela 52\% y de Chile 54\% (Ministerio de Educación Nacional, 2015)

A partir del año 2003 el MEN diseña un Sistema de Prevención y Análisis de la Deserción en las Instituciones de Educación Superior, el SPADIES (Ministerio de Educación Nacional, 2015). Así como también, ha establecido programas de fortalecimiento para desarrollar proyectos dirigidos a incrementar la permanencia estudiantil mediante convocatorias para las IES que presentan tasas de deserción altas y una mayor proporción de estudiantes vulnerables (aspectos socioeconómicos y académicos) (Ministerio de Educación Nacional, 2015). Desde el Ministerio de Educación Nacional, se han generado espacios de discusión alrededor de estos factores, buscando fomentar un proyecto educativo coherente con las exigencias de la sociedad y de los desafíos del mundo contemporáneo (Ministerio de Educación Nacional, 2009).

Diversos estudios se han realizado acerca del tema de la deserción estudiantil, es así como, en la Universidad de La Plata (Argentina), se efectuó uno que tenía como objetivo, determinar la probabilidad de abandono o graduación del alumno en la universidad y los factores personales que más se relacionaban con la duración y riesgo de cada evento. Encontraron que factores relacionados con la educación de los padres y el tipo de colegio secundario al que asistía el alumno eran importantes en la explicación de las diferencias de riesgo de deserción y probabilidades condicionales de graduación. Independientemente, de las demás características, un estudiante cuyo padre cuenta con primaria incompleta (o sin instrucción) tiene un 70\% menos posibilidades de graduarse que aquel estudiante con padre profesional (Giovagnoli, 2002).

En la Universidad del Rosario (Colombia), Lopera analiza los factores individuales, académicos y socioeconómicos que inciden sobre la probabilidad del riesgo de desertar en el programa de Economía, utilizó un modelo de duración y encontró que la deserción se produce con mayor frecuencia en los primeros semestres académicos principalmente por bajo rendimiento académico. En este sentido, el modelo de duración de riesgo proporcional en tiempo discreto, determinó los estudiantes de sexo masculino, la vinculación de los estudiantes al mercado laboral y los estudiantes provenientes de otras regiones, tienen mayor riesgo de deserción. Mientras que la edad incrementa el riesgo, pero decrece marginalmente al aumentar la misma (Lopera, 2008).

En la ciudad de Medellín se realizó un estudio en Instituciones de Educación Superior de Medellín e identificó los determinantes de la deserción en distintos grupos poblacionales y encontró diferencias entre los tres niveles de formación, las variables socioeconómicas, individuales, las relacionadas con las áreas de conocimientos y los tipos de apoyos tienen efectos diferentes en cada nivel (Aleans, 2012). En Valledupar como resultado de un Convenio entre el MEN y la Universidad Popular del Cesar, se desarrolló un estudio para caracterizar y determinar el perfil del estudiantes y encontraron que la tasa de deserción era de 45,64\%, lo cual comparada con las otras instituciones de Colombia de carácter público se encuentra en condiciones favorables (Escorcia, 2013). Sin embargo, estas tasas se regulan de manera natural, es decir, factores externos como las becas Fedecesar, el número de estudiantes admitidos, y otras variables hacen que estas tasas no aumenten. 
Para la Universidad de Cartagena, ha sido una preocupación permanente el tema de la deserción estudiantil en atención a las repercusiones sociales y económicas que implica. Desde el año de 1986 se comenzó a realizar de manera aislada acciones de fomento a la permanencia estudiantil. En el año 2011, la Institución suscribió un convenio de acompañamiento con el MEN, que permitió que la Universidad adelantara un ejercicio de articulación de las estrategias de fomento a la permanencia que se tradujeron en cuatro componentes del Sistema Integrado de Retención Estudiantil (SIRE): académico, psicosocial, socioeconómico e información, seguimiento y evaluación. Para el segundo periodo del año 2017, la Universidad de Cartagena la denominación por Sistema Institucional de Retención Estudiantil.

En cumplimiento de la Política Institucional de Responsabilidad Social, el SIRE hizo parte de los objetivos estratégicos de bienestar estudiantil. Ejercicio que incluyó la participación, de la División de Calidad, la Vicerrectoría Académica, la Oficina de Planeación, el Centro de Admisiones, el área de Registro y Control Académico, la División de Sistemas y la División de Comunicaciones y Relaciones Públicas (Universidad de Cartagena, 2012). En este sentido, la permanencia de los estudiantes en el sistema educativo se convierte en uno de sus objetivos estratégicos a partir del cual se definen alternativas de crecimiento y desarrollo, articulados con el Plan de Desarrollo Institucional 2010-2014 (Universidad de Cartagena, 2012), que posteriormente se articula al Plan de Desarrollo 2014-2018.

Para el año 2012 la Universidad de Cartagena a través de la División de Bienestar y del SIRE realizó durante el período 2009-1 a 2011-2, un diagnóstico institucional sobre factores determinantes de la deserción estudiantil en los programas de Pregrado, cuyos resultados muestran que los programas con mayor deserción fueron Matemáticas, Filosofía, Ingeniería de Alimentos, Ingeniería de Sistemas y Lingüística y Literatura. Para la cohorte que ingresó en el primer periodo de 2011 los programas que más deserción han acumulado son en orden descendente Historia, Ingeniería de Alimentos, Matemáticas y Química (Universidad de Cartagena, 2012).

Como resultado de éste, se planteó la necesidad de atender el fenómeno de la deserción estudiantil en la Universidad de Cartagena, considerando factores relacionados con el desempeño académico, los apoyos financieros y socioeconómicos, el acompañamiento psicosocial y demás beneficios estudiantiles que pueda proporcionar la institución. Para ello, se emplearon dos fuentes de información, la primera se obtuvo de la plataforma SPADIES administrada por el MEN, y la segunda fuente de información de un trabajo de campo donde se consultó a los mismos desertores (Universidad de Cartagena, 2017). Se estableció como objetivo general, el analizar la deserción estudiantil y la incidencia de los factores determinantes en estudiantes de pregrado de la Universidad de Cartagena.

\section{METODOLOGÍA}

Estudio descriptivo, correlacional, que permitió caracterizar a todos los estudiantes de pregrado de la Universidad de Cartagena, para las cohortes 2012-1 a 2016-1. Se analizará la asociación de variables para analizar la incidencia de los posibles determinantes en el riesgo de deserción estudiantil y se utilizará el Modelo Probit y el Modelo de riesgo proporcional en el tiempo que permite establecer patrones temporales de la deserción.

La población estuvo constituida por los estudiantes que ingresaron durante los períodos 2012-1 a 2016-1. La información acerca de los estudiantes que ingresaron durante estos períodos académicos se obtuvo mediante la consulta en la plataforma de SPADIES 
(Sistema de Prevención y Análisis de la Deserción en las Instituciones de Educación Superior) previa autorización de la Oficina de Planeación. Durante el período 2012-1 la población de estudiante que ingresó fue de 16.927 estudiantes.

Se obtuvo una muestra de 1547 estudiantes, los cuales representan el total de desertores de los diferentes programas presenciales y a distancia de la Universidad de Cartagena que ingresaron en la cohorte 2012-1. La muestra tuvo un error esperado del 6,5\%, una proporción esperada del 50\% y un estadístico de la distribución normal Z=1,96 al 5\% de significancia de dos colas. A partir de esta información se ponderó por proporciones de acuerdo al tamaño de cada una de los programas (Tabla 1)

Tabla 1.

Total de estudiantes que Desertaron de los programas de pregrado que ofrece la Universidad de Cartagena según modalidad y proporción de la muestra para su estudio.

\begin{tabular}{|c|c|c|c|c|}
\hline \multirow{10}{*}{ 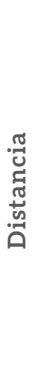 } & Programa académico & Total & Proporción & Muestra \\
\hline & Administración de Servicios de la Salud & 264 & $17,1 \%$ & 34 \\
\hline & Administración Financiera & 244 & $15,8 \%$ & 31 \\
\hline & Técnico Profesional en Operación Turística & 36 & $2,3 \%$ & 5 \\
\hline & Técnico Profesional en Procesos de Gestión Pública & 123 & $8,0 \%$ & 16 \\
\hline & Técnico Profesional en Servicios Gastronómicos & 21 & $1,4 \%$ & 3 \\
\hline & Tecnología en Administración Agropecuaria & 7 & $0,5 \%$ & 1 \\
\hline & Tecnología en Gestión Pública & 8 & $0,5 \%$ & 1 \\
\hline & Administración de Empresas & 156 & $10,1 \%$ & 20 \\
\hline & Ingeniería de Sistemas & 377 & $24,4 \%$ & 48 \\
\hline \multirow{22}{*}{ 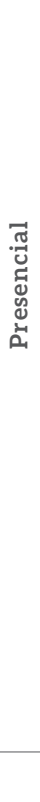 } & Administración de Empresas & 13 & $0,8 \%$ & 2 \\
\hline & Administración Industrial & 12 & $0,8 \%$ & 2 \\
\hline & Biología & 29 & $1,9 \%$ & 4 \\
\hline & Comunicación Social & 3 & $0,2 \%$ & 0 \\
\hline & Contaduría Pública & 27 & $1,7 \%$ & 3 \\
\hline & Derecho & 11 & $0,7 \%$ & 1 \\
\hline & Economía & 16 & $1,0 \%$ & 2 \\
\hline & Enfermería & 9 & $0,6 \%$ & 1 \\
\hline & Filosofía & 15 & $1,0 \%$ & 2 \\
\hline & Historia & 26 & $1,7 \%$ & 3 \\
\hline & Ingeniería Civil & 9 & $0,6 \%$ & 1 \\
\hline & Ingeniería de Alimentos & 20 & $1,3 \%$ & 3 \\
\hline & Ingeniería de Sistemas & 17 & $1,1 \%$ & 2 \\
\hline & Ingeniería Química & 12 & $0,8 \%$ & 2 \\
\hline & Lingüística y Literatura & 19 & $1,2 \%$ & 2 \\
\hline & Matemáticas & 19 & $1,2 \%$ & 2 \\
\hline & Medicina & 7 & $0,5 \%$ & 1 \\
\hline & Odontología & 8 & $0,5 \%$ & 1 \\
\hline & Química & 25 & $1,6 \%$ & 3 \\
\hline & Química Farmacéutica & 6 & $0,4 \%$ & 1 \\
\hline & Trabajo Social & 8 & $0,5 \%$ & 1 \\
\hline & Total & 1547 & $100,0 \%$ & 198 \\
\hline
\end{tabular}

Fuente: Oficina Asesora de Planeación, Universidad de Cartagena 


\section{Criterios de inclusión}

Estudiantes desertores de los programas de pregrado que ofrece la Universidad de Cartagena.

Se utilizaron dos instrumentos el Consentimiento informado que buscó la participación voluntaria del estudiante que abandona los estudios en la institución (desertor) y el instrumento de recolección de la información elaborado por los investigadores, consta de 22 preguntas. El cuestionario indaga acerca de información general, el número de preguntas es, en cuanto a información personal y familiar al momento del abandono social (2), datos relacionados con el componente motivacional para la elección de la carrera (5), situación económica (3), rendimiento académico (7), salud física y mental (3) y el componente social (2).

El presente estudio contó con el apoyo institucional, se consultó toda la información consignada en la plataforma SPADIES para la caracterización de los estudiantes y la estimación de los modelos, y se entrenó tres encuestadores para la recolección de la información de trabajo de campo a los desertores de la modalidad presencial y a distancia de los diferentes programas de la Universidad de Cartagena.

A las personas que cumplieron con los criterios de inclusión se les explicó los objetivos, se les aclaró además que podría retirarse del estudio cuando ellos así lo consideraran y se procedió a obtener el consentimiento informado. La encuesta fue subida a la plataforma de Google Drive de manera que una vez contactado el estudiante desertor la respondiera en línea. Se utilizaron las redes sociales con el fin de ubicar a las personas, el diligenciamiento de la encuesta por vía telefónica y el diligenciamiento del formato en medio físico a través de visita en sus entornos laborales o en su residencia. Terminada la recolección, los datos obtenidos fueron incorporados y analizados en una matriz creada en Microsoft Excel 2016 ․ Se utilizó el paquete estadístico STATA 14.

La información de SPADIES 2.8 fue extraída y organizada para la caracterización y estimación de los modelos de riesgo proporcional. Se estimaron los modelos de duración de riesgo proporcional con el fin de hallar los estimadores con la mayor robustez de los coeficientes y evitar problemas de varianza. El estudio se respaldó en la normatividad explicitada en la Resolución 008430 de 1993 del Ministerio de Salud de Colombia (República de Colombia 1993). Por el tipo de estudio y las técnicas a utilizar, la investigación se clasificó de riesgo mínimo para los participantes.

\section{RESULTADOS}

Para dar inicio a la caracterización de la población estudiantil, es necesario conocer que entre el periodo 2012-1 y 2016-1, el número de matriculados disminuyó considerablemente pasando de 16.927 matriculados a 15.844 (Figura 1); teniendo en promedio un descenso en el crecimiento de 0,8\%, según el reporte que genera el Sistema para la Prevención de la Deserción de la Educación Superior (SPADIES). Los periodos 2014-2 y 2015-1 han sido aquellos que han tenido tasa de crecimiento negativa con $3,1 \%$ y $3,5 \%$ respectivamente. 
Figura 1: Número de estudiantes matriculados en la Universidad de Cartagena periodo 2012-I a 2016--I

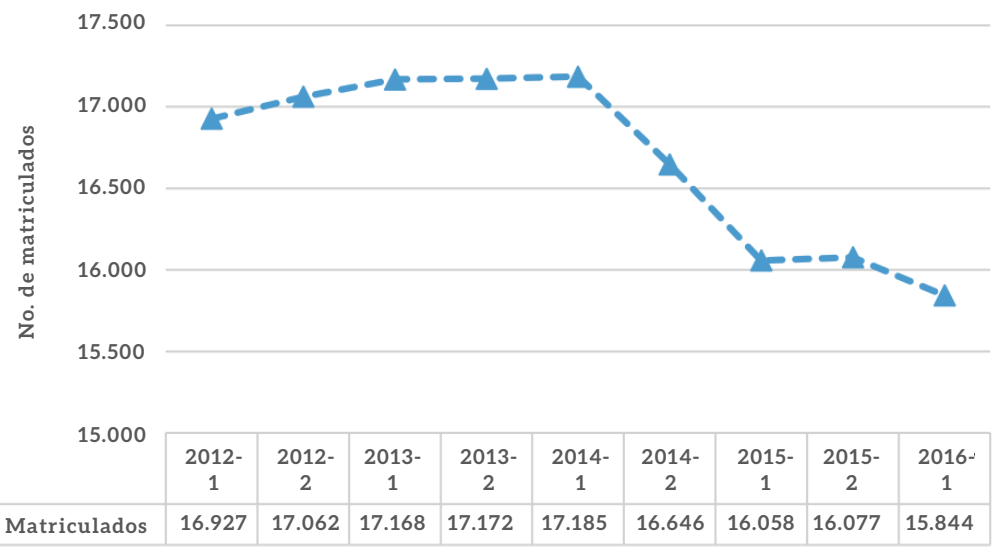

Fuente: Elaboración de los autores con base en SPADIES 2.8

A nivel general la Universidad de Cartagena ha contado en promedio entre el periodo de 2012-1 y 2016- con una distribución por sexo relativamente equilibrada. El sexo femenino en promedio representa el $56 \%$ de la comunidad estudiantil, mientras que el masculino el 44\% en promedio (Figura 2). La participación femenina en la formación profesional se ha mantenido en un 44\%, en la formación técnica 2,5\% y en la tecnológica 1,1\%, ésta ha ido descendiendo desde 2012-1 hasta estabilizarse en el 2015 y 2016-1. En cuanto al sexo masculino, su participación en promedio es 14,5 puntos porcentuales (pp) menos que el femenino para el nivel universitario, mientras que para el técnico y tecnológico es $2,1 \%$ y $0,7 \%$ respectivamente.

Figura 2: Distribución de estudiantes matriculados por género en la Universidad de Cartagena periodo 2012-I a 2016-I

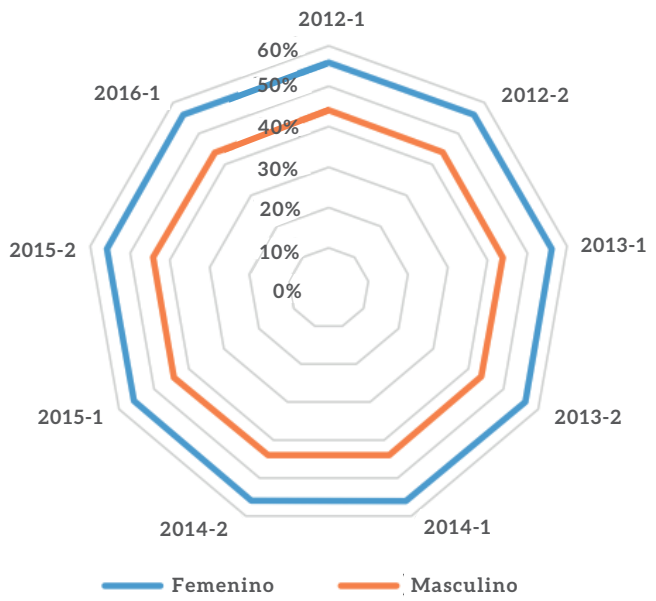

Fuente: Elaboración de los autores con base en SPADIES 2.8 
Por área del conocimiento, los programas de Economía, Administración, Contaduría $\mathrm{y}$ afines es donde mayor presencia tiene el sexo femenino, con un promedio para el periodo analizado de 32,9\%, al igual que para el masculino con 19,4\% (Figura 3). Las áreas de Agronomía y ciencias de la educación, el porcentaje de matriculados es menor, por lo que su representatividad y diferenciación por sexo es imperceptible en términos porcentuales.

Figura 3: Participación promedio por género de acuerdo al área de conocimiento. Promedio 2012-I a 2016-I

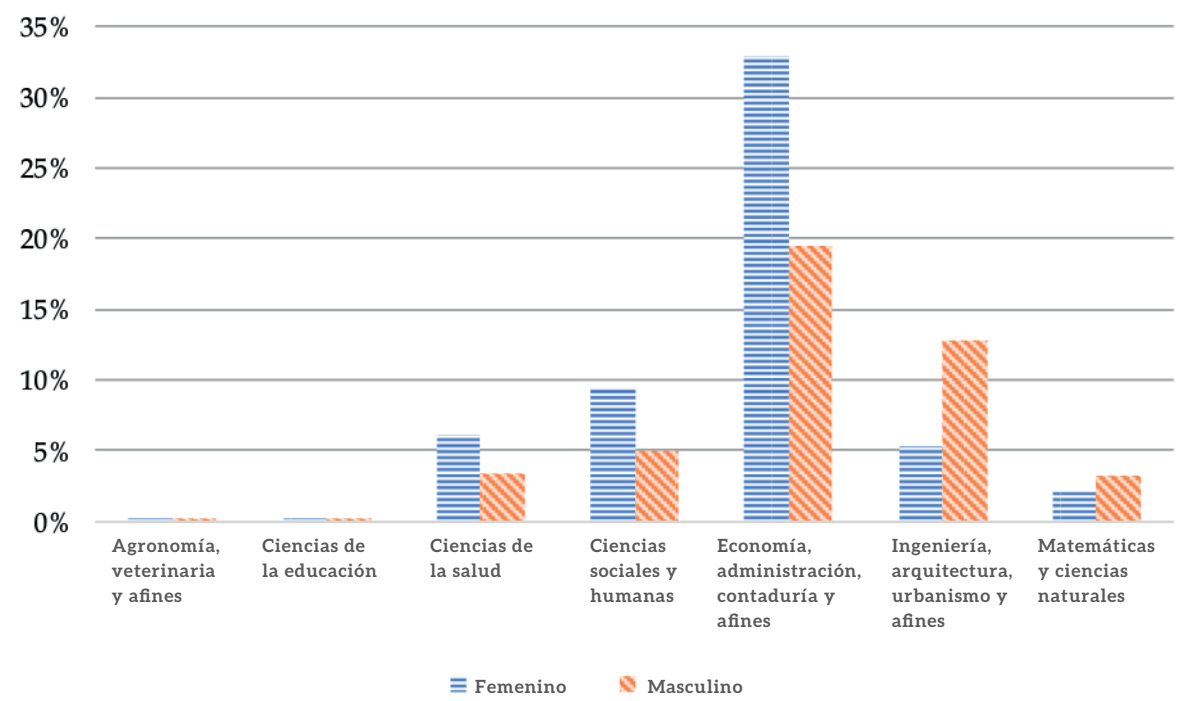

Fuente: Elaboración de los autores con base en SPADIES 2.8

La Figura 4 muestra que el 52\% de los estudiantes matriculados en Universidad de Cartagena en promedio, pertenecen al estrato socioeconómico 1, seguido del estrato 2 con 21\%. En el periodo 2013-2 se muestra un comportamiento atípico donde el estrato 1, 3, 4 y 5 aumentan su participación, siendo el primero en una mayor proporción, reduciendo en 87,5\% respecto al periodo anterior la participación del estrato 2 . 
Figura 4: Proporción de estudiantes por estrato socioeconómico periodo 2012-I a 2016-I

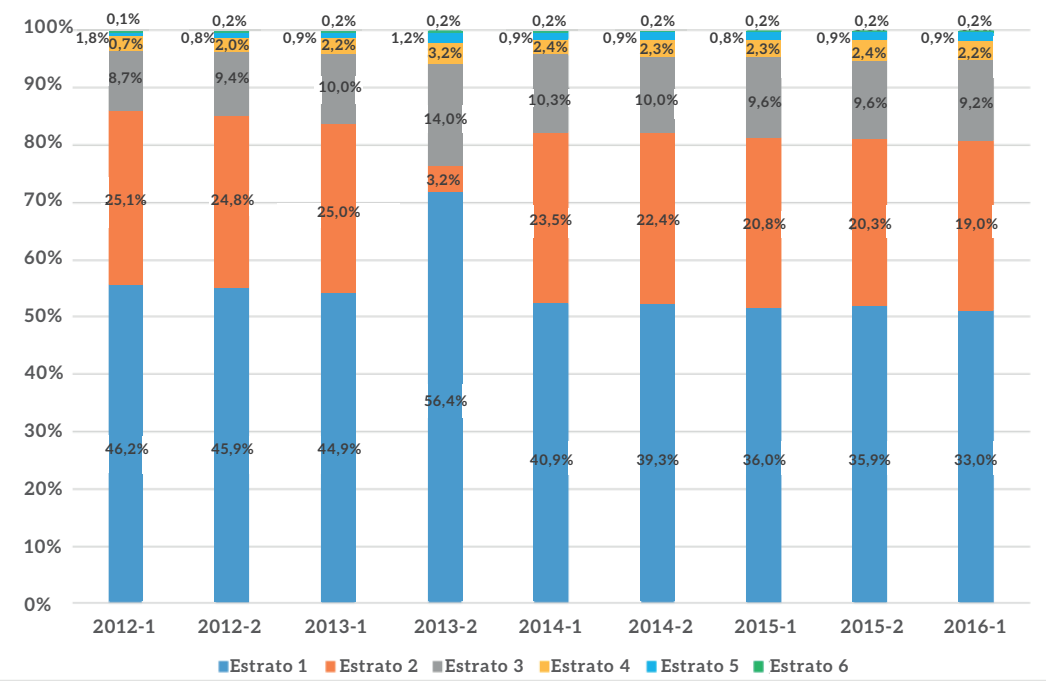

Fuente: Elaboración de los autores con base en SPADIES 2.8

El número de matriculados cuya Madre tiene un nivel educativo de básica secundaria se ha mantenido en promedio con un 35,2\% en los periodos analizados (Figura 5), sin embargo la tasa de crecimiento de los matriculados cuyo nivel educativo de la madre es básica primaria o inferior ha ido disminuyendo, situación planteada en los estudios realizados por el MEN (4). El porcentaje de matriculados cuya madre tienen nivel universitario o superior mostró una tendencia creciente hasta el 2014-2.

Figura 5: Distribución de estudiantes de acuerdo al nivel de formación de la madre periodo 2012-I a 2016-I

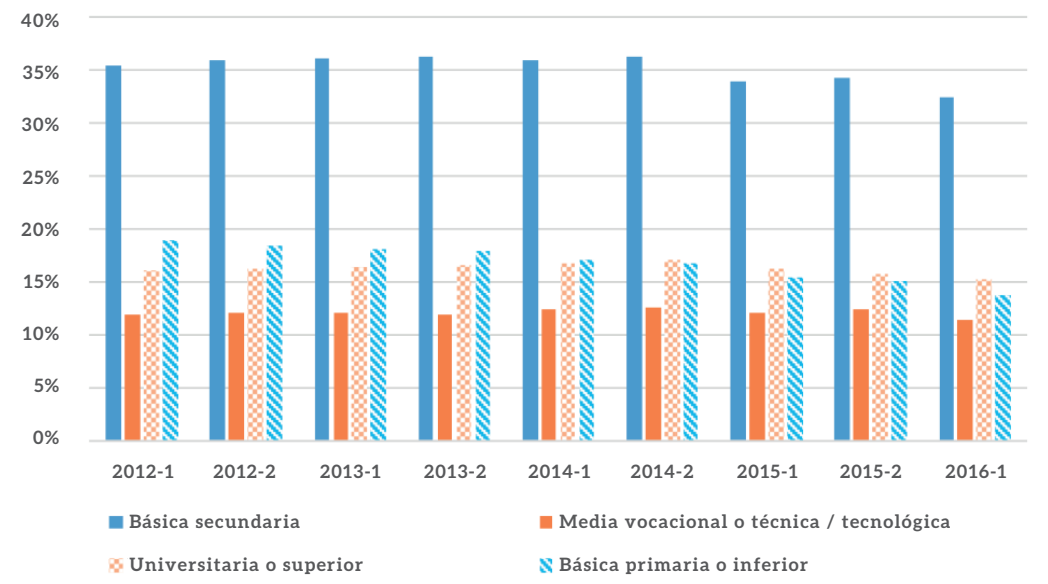

Fuente: Elaboración de los autores con base en SPADIES 2.8 
En las pruebas de estado, el 54,7\% de los estudiantes matriculados en la Universidad de Cartagena se ubican en promedio en la clasificación de Bajo y Medio (Figura 6). Solo el $20 \%$ en promedio clasifica en alto. Todas las clasificaciones presentan una tendencia a la baja, sin embargo el nivel bajo es quien en el periodo analizado ha tenido mayor disminución al pasar de $31,2 \%$ a $21,1 \%$.

Figura 6: Distribución de estudiantes de acuerdo al nivel alcanzado en la prueba Saber 11 periodo 2012-I a 2016-I

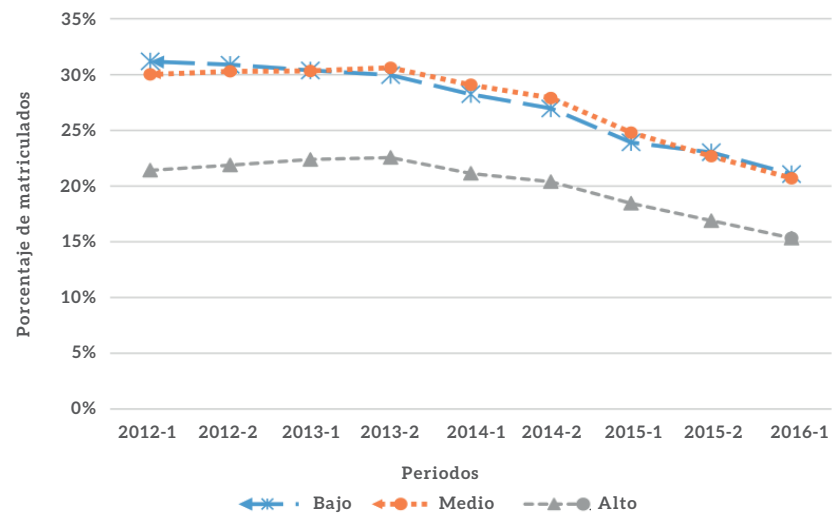

La tasa de repitencia está definida como la cantidad de materias perdidas sobre materias vistas del periodo anterior. En ese sentido, la mayor tasa de repitencia para estudiantes matriculados en el periodo analizado en la Universidad de Cartagena se encuentran entre el $0 \%$ al 10\%. El periodo 2013-2 el 96,8\% de los estudiantes se encontraba en la tasa más baja de repitencia, a diferencia del periodo anterior (20131) en la $33,7 \%$ de los estudiantes matriculados tenían la tasa de repitencia más alta (100\%). En el periodo 2014-2, el 55,3\% de los estudiantes tenían una tasa de repitencia entre el 10 y $20 \%$ (Figura 7).

Figura 7: Proporción de estudiantes de acuerdo a la tasa de repitencia periodo 2012-I a 2016-I

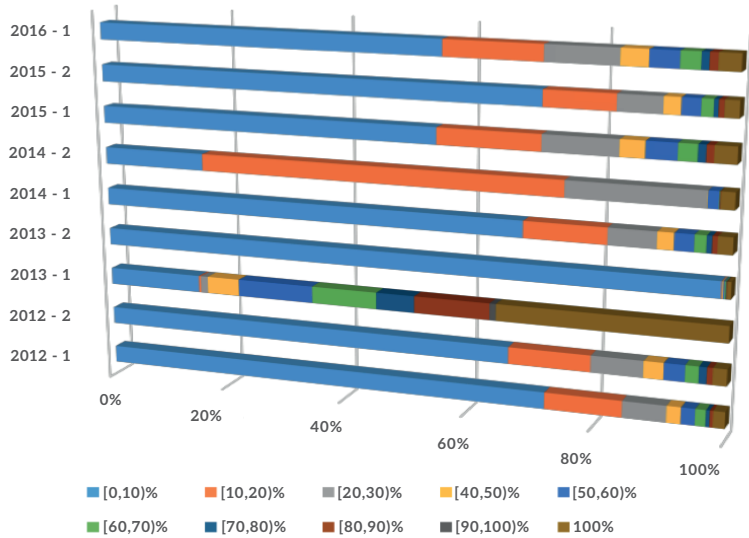

Fuente: Elaboración de los autores con base en SPADIES 2.8 


\section{Caracterización de la población desertora 2012-I a 2016-I Universidad de Cartagena}

En SPADIES se reporta que la deserción en la Universidad de Cartagena en las cohortes 2012-I y 2016-I (periodo de análisis) hay una tendencia decreciente pasando de 15\% aproximadamente en 2012-I a 11\% en 2016-I, lo cual representa una reducción de 5 (pp). Por género, la población femenina ha mantenido una tasa de deserción menor a la de los hombres, presentándose una brecha, en promedio, de 2 pp (Figura 8).

Figura 8: Tasa de deserción por género en los estudiantes de la Universidad de Cartagena. 2012-I a 2016-I

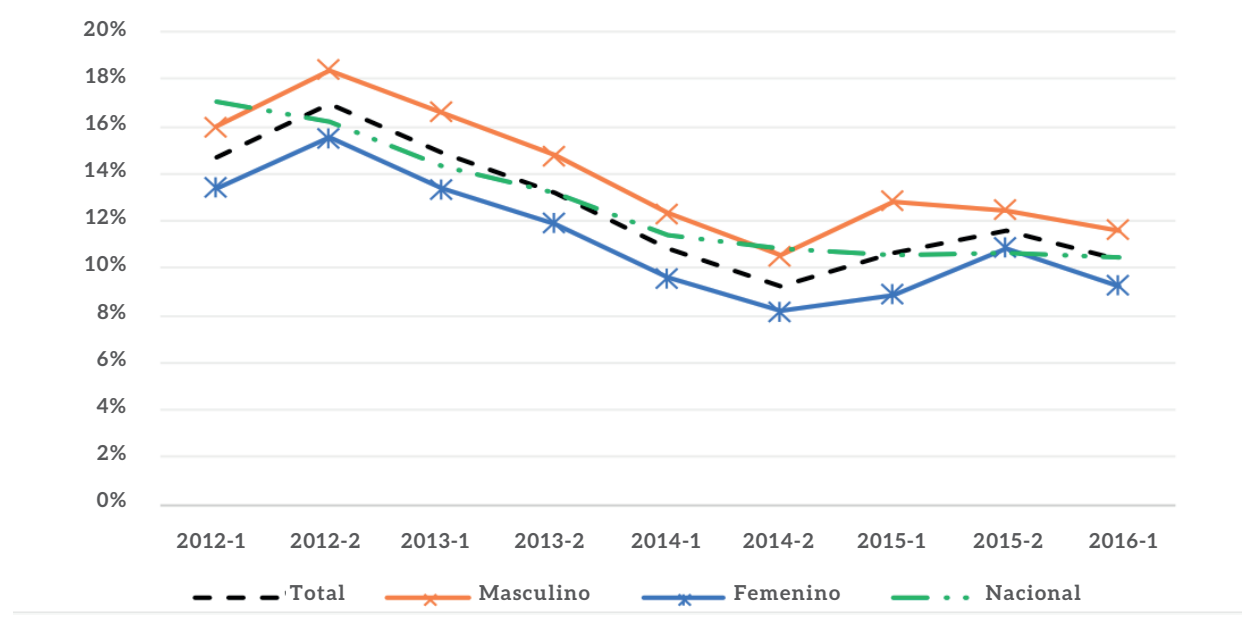

Fuente: Elaboración de los autores con base en SPADIES 2.8

Según la información de la Figura 9, la tasa de deserción de los programas a distancia de la Universidad de Cartagena ha sido superior que la tasa de los programas presenciales, traduciéndose en una brecha de casi 4 pp en promedio en el periodo de estudio. Esta tasa de deserción ha disminuido cerca de $4 \mathrm{pp}$ aproximadamente en la modalidad a distancia y presencial.

De acuerdo a información proporcionada por la Oficina de Admisiones, Registro y Control de la Universidad, los programas a Distancia se adscribieron a las diferentes Facultades por medio de resoluciones de rectoría del 21 de diciembre de 2012. Esta decisión estaba orientada a fortalecer la modalidad a distancia. Se tomó además la decisión de reducir de oferta de los programas en algunos centros tutoriales y Ceres y se establecieron criterios de ingreso comunes a los exigidos en los programas presenciales, ocasionando una disminución progresiva en el número de estudiantes matriculados en esta modalidad. En el periodo 2015-2, se presenta un paro estudiantil principalmente en los programas de pregrado, trayendo consigo un gran aumento de aplazamientos (133 en total).

Contrastando la evolución de la tasa de deserción de la Universidad con el promedio 
nacional, los comportamientos han sido similares, lo que indica que ha ido en concordancia a las directrices y metas propuestas a nivel nacional. Para el año 2014 presentó un promedio menor a las cifras nacionales.

Figura 9: Tasa de deserción por modalidad en la Universidad de Cartagena. 2012-I a 2016-I

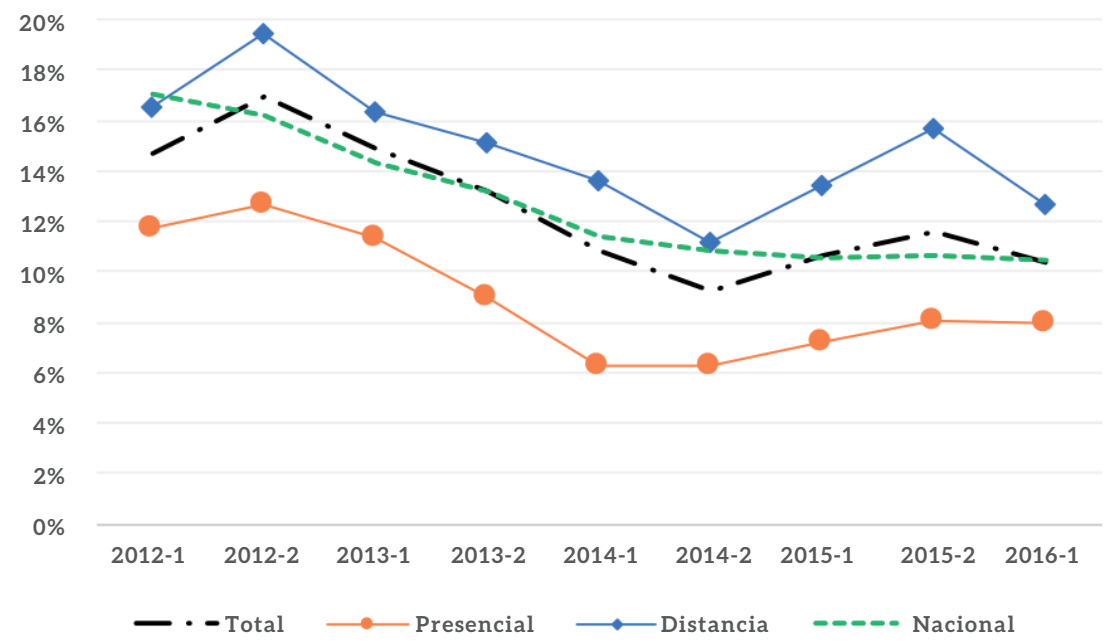

Fuente: Elaboración de los autores con base en SPADIES 2.8

En cuanto a la deserción por género en cada una de las modalidades, se corrobora nuevamente que las mujeres desertan menos que los hombres. Sin embargo, cohortes como 2014-II para los programas presenciales y 2015-II para la metodología a distancia, la diferencia entre la tasa de los hombres y las mujeres disminuyó por lo que no se observa mucha diferencia en estos periodos (Figura 10). Por otro lado, las diferencias por género son más marcadas en los programas a distancia con brechas de hasta 5 pp, como en el periodo 2012-I y 2015-I.

Abordando la deserción por programas en cada una de las modalidades, la Figura 10 evidencia que los programas de Matemáticas, Ingeniería de Sistemas, Filosofía, Biología, Historia y Química han tenido tasas de deserción mayores al 15\%. El programa de Enfermería que presenta una tasa de deserción masculina cercana al 17\%. Los programas que han mantenido una tasa de deserción por debajo del 10\% son: Ingeniería Civil, Química Farmacéutica, Medicina, Administración Industrial, Trabajo Social, Derecho, Contaduría Pública, Comunicación Social, Enfermería e Ingeniería Química. Finalmente, cabe destacar que el único programa presencial que ha mantenido una cifra por debajo del 5\% es el programa de Odontología. 
Figura 10: Tasa de deserción en los programas presenciales de la Universidad de Cartagena. Promedio 2012-I a 2016-I.

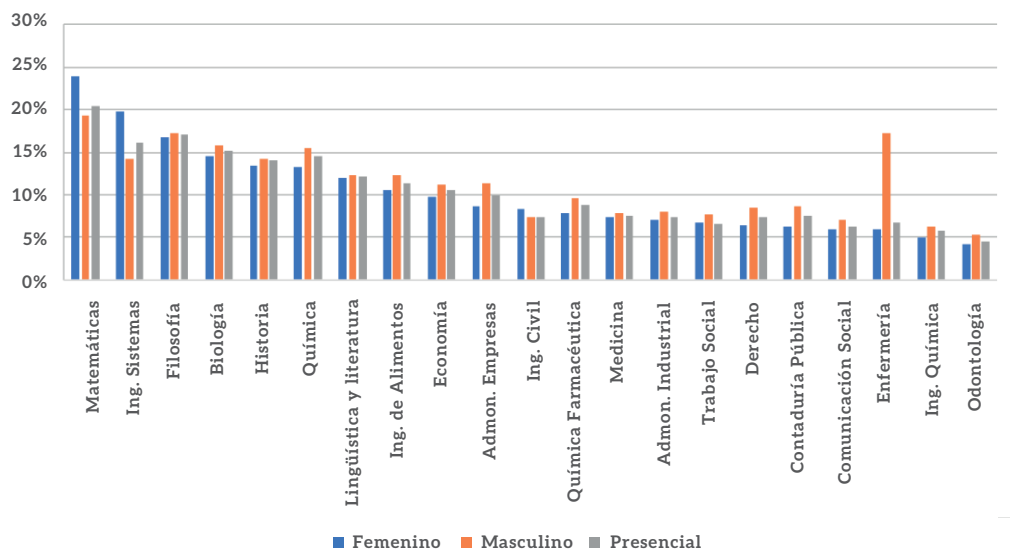

Fuente: Elaboración de los autores con base en SPADIES 2.8

La Figura 11 muestra que durante el periodo de estudio la tasa de deserción en la población masculina y la población femenina que trabajaba, como la que no trabaja al momento de presentar la prueba de Estado, tuvieron un comportamiento decreciente y constante. Por su parte, las mujeres que si trabajaban al momento de presentar la prueba el comportamiento de su tasa de deserción fue un tanto volátil, dado que en 2012-I y 2012-II esta fue igual o superior a la de los hombres que no trabajaban, luego tuvo un comportamiento creciente hasta superar la tasa de deserción en 2014-I de los hombres que, si trabajaban, y finalmente decrece siendo la menor tasa comparada a las otras tres categorías en 2016-I. Es de señalar, que al final del periodo de estudio las tasas de deserción tienden a converger para los hombres que trabajaban y que no trabajaban junto con las mujeres que no trabajaban al momento de presentar la prueba Saber 11

Figura 11: Tasa de deserción por género de los estudiantes que trabajaban o no al momento de presentar la prueba Saber 11. Periodo 2012-I a 2016-I

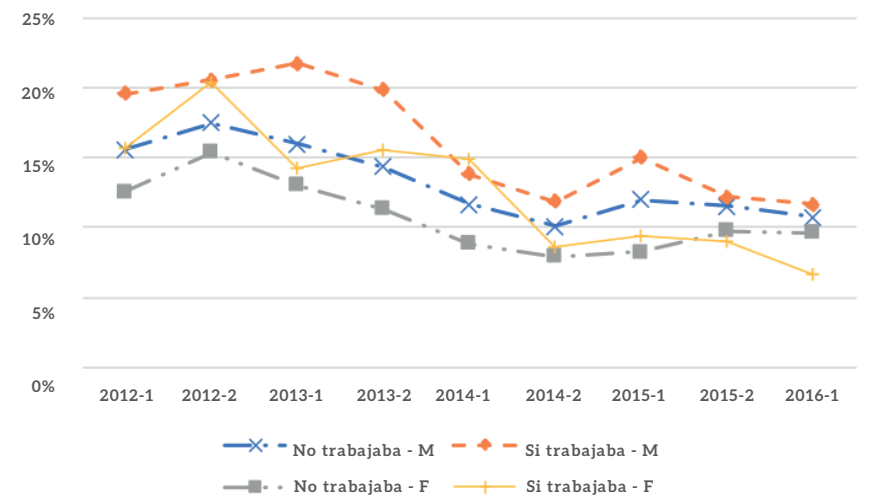


En relación al nivel educativo alcanzado por la madre de cada uno de los estudiantes de pregrado de la Universidad de Cartagena, (Tabla 2), los estudiantes de los programas a distancia presentan mayores tasas de deserción que los estudiantes presenciales. En las primeras tres cohortes de los niveles de educación se evidencian cifras de dos dígitos para ambas modalidades, pero a partir de 2013-I y 2014-I, la tasa de deserción de los estudiantes presenciales de acuerdo al nivel de formación de sus madres se sitúa por debajo del 10\% en la mayoría de los casos, a excepción de básica primaria para la cohorte 2016-I. Finalmente, en esta variable las brechas entre las tasas de deserción son amplias, puesto que en básica primaria es de 6,2 pp, básica secundaria $5,2 \mathrm{pp}$, media técnica vocacional $6,4 \mathrm{pp}$, y universitario $6 \mathrm{pp}$ en promedio durante todo el periodo.

Tabla 2.

Tasa de deserción por nivel educativo de la madre por modalidad en los estudiantes de la Universidad de Cartagena. Periodo 2012-I a 2016-I

\begin{tabular}{|c|c|c|c|c|c|c|c|c|c|}
\hline Nivel educativo de la madre & $2012-1$ & | 2012-2 & 2013-1 | & $|2013-2|$ & 2014-1 & 2014-2 & $2015-1$ & | 2015-2 & | 2016-1 \\
\hline \multicolumn{10}{|l|}{ Básica primaria o inferior } \\
\hline Distancia & $15,2 \%$ & $20,2 \%$ & $18,6 \%$ & $17,4 \%$ & $12,7 \%$ & $11,7 \%$ & $15,4 \%$ & $17,2 \%$ & $13,2 \%$ \\
\hline Presencial & $9,9 \%$ & $12,9 \%$ & $12,6 \%$ & $10,4 \%$ & $6,8 \%$ & $6,4 \%$ & $7,6 \%$ & $8,3 \%$ & $11,2 \%$ \\
\hline \multicolumn{10}{|l|}{ Básica secundaria } \\
\hline Distancia & $14,8 \%$ & $19,0 \%$ & $15,9 \%$ & $15,3 \%$ & $13,2 \%$ & $10,4 \%$ & $12,0 \%$ & $14,9 \%$ & $12,3 \%$ \\
\hline Presencial & $12,0 \%$ & $12,4 \%$ & $9,7 \%$ & $9,3 \%$ & $6,8 \%$ & $7,1 \%$ & $7,6 \%$ & $7,6 \%$ & $8,3 \%$ \\
\hline \multicolumn{10}{|c|}{ Media vocacional o técnica/tecnológica } \\
\hline Distancia & $16,9 \%$ & $20,7 \%$ & $14,0 \%$ & $14,7 \%$ & $15,3 \%$ & $10,8 \%$ & $14,3 \%$ & $13,0 \%$ & $13,2 \%$ \\
\hline Presencial & $11,7 \%$ & $10,5 \%$ & $11,1 \%$ & $7,8 \%$ & $6,4 \%$ & $5,5 \%$ & $7,5 \%$ & $7,3 \%$ & $7,3 \%$ \\
\hline \multicolumn{10}{|l|}{ Universitaria o superior } \\
\hline Distancia & $21,0 \%$ & $21,4 \%$ & $13,9 \%$ & $12,0 \%$ & $14,2 \%$ & $11,4 \%$ & $10,4 \%$ & $11,5 \%$ & $10,9 \%$ \\
\hline Presencial & $12,5 \%$ & $12,3 \%$ & $12,6 \%$ & $7,3 \%$ & $4,1 \%$ & $4,9 \%$ & $5,5 \%$ & $6,7 \%$ & $6,6 \%$ \\
\hline
\end{tabular}

Fuente: Elaboración de los autores con base en SPADIES 2.8

\section{Prueba saber 11 y la deserción de los estudiantes de pregrado}

En cuanto al análisis de la deserción y clasificación de las pruebas Saber 11 por modalidad, la Figura 13 muestra que durante todo el período de análisis aquellos que obtuvieron una clasificación baja o media en las pruebas Saber 11 y que pertenecen a la metodología a distancia, en promedio, fueron quienes presentaron mayores tasas de deserción. Cabe recordar que, los periodos de 2012-2 y 2015-2 tuvieron ciertas situaciones coyunturales que hicieron elevar las cifras de deserción siendo los estudiantes con características socioeconómicas más complejas los más vulnerables frente a esta problemática.

En ese sentido, los estudiantes que pertenecen a la modalidad a distancia son quienes presentan mayores cifras de deserción y en los que mayormente se deben poner los esfuerzos para garantizar su continuidad. Dado que, aún aquellos que muestran una clasificación alta en las pruebas Saber 11, tienen tasas de deserción mayores que quienes obtuvieron clasificación media e incluso baja en los programas de pregrado, tal y como se evidencia en los periodos 2015-I y 2015-II (Figura 12). 
En relación a la modalidad presencial, en todos los periodos es claro que existe una relación inversa entre la tasa de deserción y la clasificación obtenida en las pruebas Saber 11, es decir, entre más baja es la clasificación mayor será la tasa de deserción de acuerdo a lo que se observa en la Figura 12.

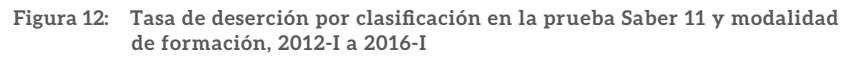
de formación, 2012-I a 2016-I

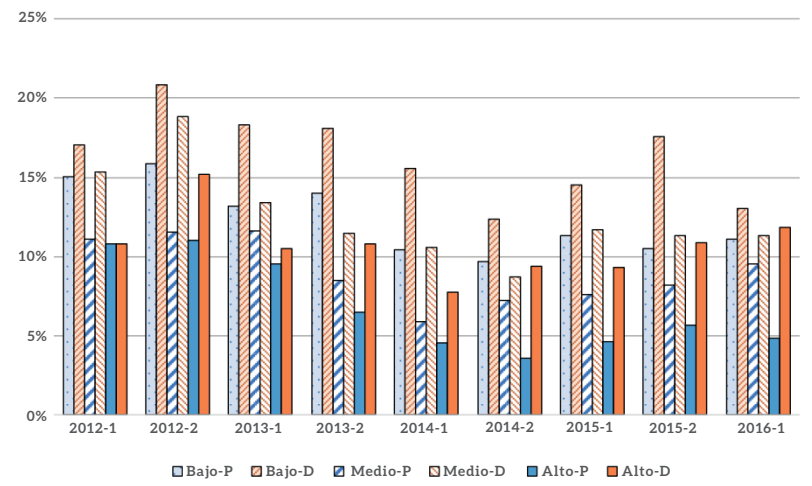

Fuente: Elaboración de los autores con base en SPADIES 2.8

Contrastando estas variables por género, la tendencia permanece en cuanto a lo que se ha mostrado anteriormente, los hombres son quienes presentan mayores problemas en temas de deserción. La Figura 13, evidencia que la población masculina con baja clasificación en las pruebas Saber 11, da a conocer las mayores cifras a lo largo de todo el periodo de estudio con tasas hasta del $20 \%$, que comparado con la población femenina con clasificación alta la brecha, en promedio, es de casi 10 pp en todos los periodos.

Es de recalcar, que la población femenina con clasificación baja y media ha presentado convergencia con la población masculina con clasificación media y alta, respectivamente. Lo cual da muestra que las clasificaciones en estas pruebas estandarizadas no implican del todo el hecho que una persona posea mayor probabilidad de desertar, es decir, existen otras características asociadas a este hecho.

Figura 13: Tasa de deserción por género y clasificación en la prueba SABER 11 y modalidad de formación, periodo 2012-I a 2016-I

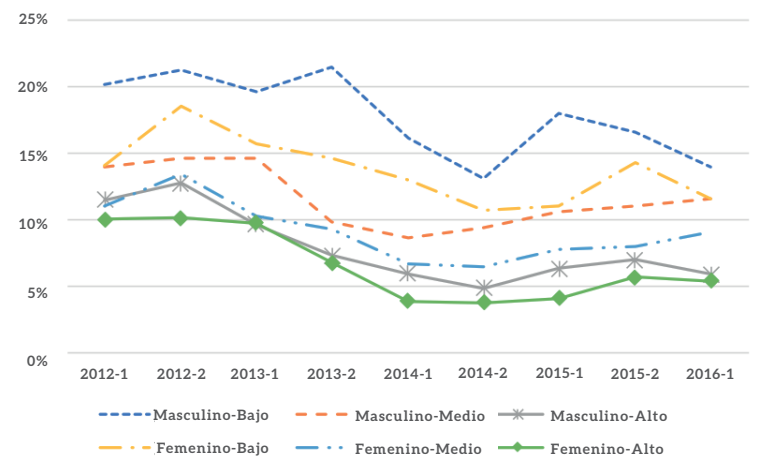

Fuente: Elaboración de los autores con base en SPADIES 2.8 
De acuerdo a la clasificación de la deserción sugerida por el MEN en temprana y tardía. Teniendo en cuenta la Figura 14, se confirma lo que la literatura afirma en muchos casos, y es que la mayor parte de la deserción se da en los primeros cuatro semestres. Para el caso de la Universidad de Cartagena, analizando la población que ingresó en el periodo 2012-I, alrededor del 80\% de los desertores se encontraban en los primeros semestres, siendo el primer periodo el más crítico. Sin embargo, es importante anotar que resta un $20 \%$ que al final de la carrera también desertaron.

En cuanto a los programas presenciales, en términos cuantitativos los que mayores desertores presentaron fueron Biología (29), Contaduría Pública (27), Historia (26), Química (25) e Ingeniería de Alimentos (20). Además, haciendo el análisis por deserción temprana y tardía, se observa que los programas de Ingeniería Civil, Ingeniería de Sistemas, Biología, Filosofía y Administración de empresas, presentan los valores más altos en deserción temprana. Finalmente, respecto a la deserción tardía los programas de Historia, Lingüística y Literatura, Enfermería y Economía, son los que presentan mayores cifras dentro de esta clasificación, en ese orden.

Figura 14: Distribución de los desertores por semestre. Universidad de Cartagena, Periodo 2012-I a 2016-I

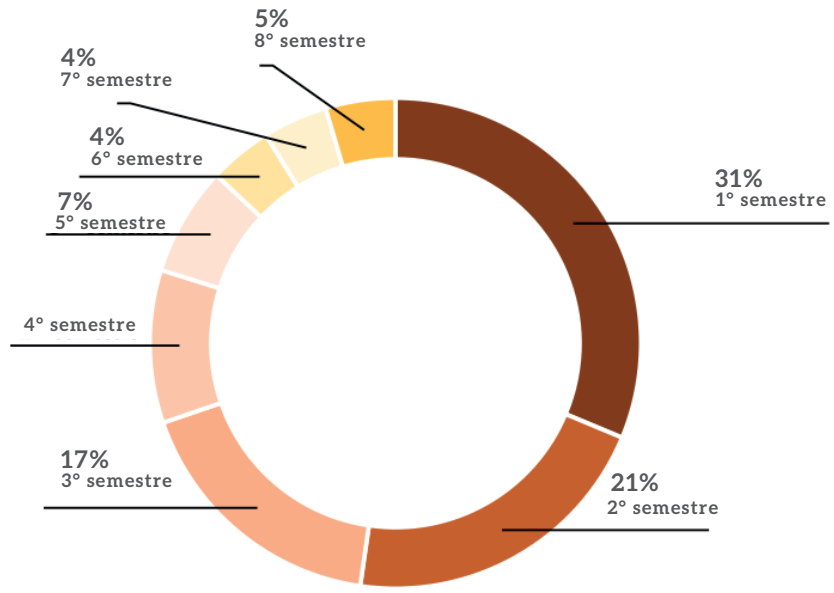

Fuente: Elaboración de los autores con base en SPADIES 2.8

Diferenciando por la modalidad de estudio en el análisis de los estudiantes que ingresaron en 2012-I. La Tabla 3, presenta que durante todos los periodos la proporción de desertores de la metodología a distancia fue superior a los presenciales, acortándose esta brecha en el periodo 2015-II cuando existe menor probabilidad de desertar. Asimismo, la tasa de deserción para cada periodo muestra que para ambas modalidades hubo más deserción en los cuatro primeros semestres $82 \%$ para distancia y $73 \%$ presencial, respectivamente. Cabe resaltar que, en modalidad presencial la proporción de desertores de últimos semestres supera en términos relativos a la proporción de distancia. 
Tabla 3.

Evolución de la deserción por metodología de formación en la Universidad de Cartagena. Cohorte 2012-I

\begin{tabular}{lrrrrr|r|r|r|r|r}
\hline Estado & 2012-1 & 2012-2 & 2013-1 & 2013-2 & 2014-1 & 2014-2 & 2015-1 & 2015-2 & TOTAL \\
\hline N ${ }^{\circ}$ Desertores & 484 & 325 & 270 & 156 & 111 & 64 & 66 & 71 & 1547 \\
Distancia & 409 & 272 & 207 & 121 & 94 & 52 & 44 & 37 & 1236 & 311 \\
Presencial & 75 & 53 & 63 & 35 & 17 & 12 & 22 & 34 & $79,9 \%$ \\
Distancia (\%) & $84,5 \%$ & $83,7 \%$ & $76,7 \%$ & $77,6 \%$ & $84,7 \%$ & $81,3 \%$ & $66,7 \%$ & $52,1 \%$ & $20,1 \%$ \\
Presencial (\%) & $15,5 \%$ & $16,3 \%$ & $23,3 \%$ & $22,4 \%$ & $15,3 \%$ & $18,8 \%$ & $33,3 \%$ & $47,9 \%$ & & \\
& & & & & & & & & & \\
Deserción (\%) & $31,3 \%$ & $21,0 \%$ & $17,5 \%$ & $10,1 \%$ & $7,2 \%$ & $4,1 \%$ & $4,3 \%$ & $4,6 \%$ & $100 \%$ \\
Distancia & $33,1 \%$ & $22,0 \%$ & $16,7 \%$ & $9,8 \%$ & $7,6 \%$ & $4,2 \%$ & $3,6 \%$ & $3,0 \%$ & $100 \%$ \\
Presencial & 24,1 & $17,0 \%$ & $20,3 \%$ & $11,3 \%$ & $5,5 \%$ & $3,9 \%$ & $7,1 \%$ & $10,9 \%$ & $100 \%$
\end{tabular}

Fuente: Elaboración de los autores con base en SPADIES 2.8

\section{Modelos de duración de riesgo proporcional}

En las funciones presentadas en la sección anterior, se observa cómo va disminuyendo la probabilidad de permanecer o caer en cierto estado asociado al tiempo. Sin embargo, el tiempo no es la única variable relevante para estudiar este hecho, por lo que se hace necesario incorporar otras variables de carácter explicativo relevantes en la probabilidad de desertar o no. Es por ello que se utilizan los modelos de riesgo proporcional, donde la variable dependiente para este caso es el riesgo a desertar o no, y como variables independientes el tiempo y un conjunto de variables explicativas asociadas a características socioeconómicas, académicas e institucionales.

$$
h(t)=h_{0}(t) * \exp \left(\beta_{0}+\beta_{1} X_{1}\right)
$$

Donde, $h_{0}(t)$ captura el efecto del tiempo y el resto de la expresión corresponde al conjunto de variables explicativas o grupo de control. Que para el caso de este estudio se incluyeron las siguientes variables (Tabla 4):

Tabla 4.

Variables incluidas en el modelo de duración. Universidad de Cartagena. Periodo 2012-I a 2016-I

\begin{tabular}{lcc}
\hline Variable dependiente & Descripción & Fuente \\
\hline Riesgo de desertar & Desertores = 1, Otro caso = & SPADIES 2.8 \\
\hline Variable independientes & Descripción & Fuente \\
\hline Género & Hombres = 1, Mujeres = 0 & SPADIES 2.8 \\
\hline $\begin{array}{l}\text { Trabaja al momento de } \\
\text { presentar las pruebas SABER 11 }\end{array}$ & $\mathrm{Si}=1$, No $=0$ & SPADIES 2.8 \\
\hline Modalidad & Distancia = 1, Presencial = 0 & SPADIES 2.8
\end{tabular}


Tabla 4.

Variables incluidas en el modelo de duración. Universidad de Cartagena. Periodo 2012-I a 2016-I

\begin{tabular}{|c|c|c|}
\hline Variable independientes & Descripción & Fuente \\
\hline Nivel educativo de la madre & Primaria $=1$, Otro caso $=0$ & SPADIES 2.8 \\
\hline Ingresos familiares & Entre 0 y 1 salario $=1$, Otro $=0$ & SPADIES 2.8 \\
\hline Clasificación pruebas Saber 11 & Bajo $=1$, Otro caso $=0$ & SPADIES 2.8 \\
\hline $\begin{array}{l}\text { Edad al momento de presentar } \\
\text { las pruebas Saber } 11\end{array}$ & 26 o más $=1$, Otro caso $=0$ & SPADIES 2.8 \\
\hline $\begin{array}{l}\text { Número de apoyos } \\
\text { académicos recibidos }\end{array}$ & Ninguno $=1$, Otro caso $=0$ & SPADIES 2.8 \\
\hline $\begin{array}{l}\text { Número de apoyos } \\
\text { financieros recibidos }\end{array}$ & Ninguno $=1$, Otro caso $=0$ & SPADIES 2.8 \\
\hline Estado socioeconómico & Estrato $1=1$, Otro caso $=0$ & SPADIES 2.8 \\
\hline Tasa de repitencia & Mayor a $50 \%=1$, Otro caso $=0$ & SPADIES 2.8 \\
\hline Tamaño de la familia & 10 a 12 personas $=1$, Otro $=0$ & SPADIES 2.8 \\
\hline
\end{tabular}

Fuente: Elaboración de los autores con base en SPADIES 2.8

\section{Modelo de riesgo proporcional}

Las estimaciones para el total de la Universidad de Cartagena (Tabla 5), en la que la unidad de análisis correspondió a los estudiantes que ingresaron en la cohorte 2012-I, y a la cual mediante el modelo se hizo el respectivo seguimiento para determinar el nivel de riesgo de desertar.

Abordando el análisis, se tiene que efectivamente como lo expresa la literatura son los hombres quienes mayor riesgo de desertar presentan, en promedio, en un $27.8 \%$ en comparación con las mujeres. Asimismo, quienes trabajaban al momento de presentar las pruebas Saber 11 acumulan un riesgo de desertar de 35.2\% comparados con los que no trabajaban. En ese mismo sentido, aquellos estudiantes que pertenecen a la modalidad de enseñanza a distancia acumulan un riesgo de desertar $20.8 \%$ mayor a los que se encuentran en la modalidad presencial. Con esto se confirma el hecho que ser hombre y estudiar en algún programa a distancia, aumenta el riesgo de desertar de manera significativa.

Por otro lado, midiendo la influencia del nivel de educación alcanzado de la madre se halló que, aunque los parámetros no fueron significativos, el resultados de los coeficientes fue el esperado por lo que se interpreta que aquellos estudiantes con madres que hayan alcanzado nivel de secundaria, técnico/tecnológico y universitario presentan un riesgo menor de desertar en $0.1 \%, 13.8 \%$ y $14.1 \%$. En relación a los ingresos familiares, los coeficientes no fueron en su mayoría significativos dado 
que la distribución de los ingresos entre los estudiantes es similar, es decir, no existen marcadas diferencias entre los ingresos al interior del núcleo familiar de la gran mayoría de los estudiantes. Asimismo, ocurre con la variable de estrato socioeconómico, dado que la mayoría de los estudiantes pertenecen a los estratos 1,2 y 3 , por lo que no existe diferencia estadística al momento del análisis.

En la clasificación obtenida en las pruebas Saber 11, esta variable guarda una alta correlación con el riesgo de desertar, dado que aquellos estudiantes con clasificación media y alta en estas pruebas estandarizadas presentan un riesgo menor a los que tienen clasificación baja en $24.7 \%$ y $21 \%$, respectivamente. En ese mismo sentido, la edad es un determinante importante para medir el riesgo, dado que aquellos estudiantes con edad entre los 16 y 25 años presentan un riesgo menor entre $16 \%$ y $34 \%$ de desertar, frente a aquellos que tienen una edad igual o superior a los 26 años. Dentro del grupo de variables institucionales, encontramos que sus coeficientes fueron altamente significativos para la explicación del riesgo de desertar, dado que un estudiante que recibe entre 1 y 3 apoyos académicos tiende a disminuir su riesgo de desertar entre $8 \%$ y casi un 100\%, en comparación a aquellos que no reciben ningún tipo de ayuda académica. No obstante, si se supera este número de apoyos su riesgo de desertar aumenta en un 37\%, en lugar de no recibir ningún apoyo. Es decir, es mucho más grave brindar muchos apoyos en lugar de no darlos.

Ahora bien, en los apoyos financieros ocurre algo similar con el número de apoyos entre 1 y 3, y los que reciben más de 4 apoyos. Para el primer caso, el riesgo disminuye en casi un $100 \%$ en comparación a los que no reciben ningún tipo de ayuda, por lo que a priori se puede indicar que estos apoyos son más efectivos que los académicos. En relación a la tasa de repitencia, se observa que aquellos que tienen un porcentaje de repitencia menor al 20\%, tienen un riesgo 2 veces menor en comparación con los que presentan una tasa mayor al 50\%. Sin embargo, los que presentan una tasa entre $20 \%$ y $30 \%$ tienen un riesgo 2 veces mayor que quienes presentan una tasa mayor al $50 \%$. Lo anterior, puede ser explicado por el hecho que la mayoría de los estudiantes presentan una tasa de repitencia entre $20 \%$ y $30 \%$.

Por último, el tamaño del hogar en ninguna de sus categorías demuestra significancia estadística, por lo que no brinda una explicación adecuada dentro de los principales determinantes del riesgo a desertar. 
Tabla 5.

Modelo de duración Universidad de Cartagena, Periodo 2012-I a 2016-I

\begin{tabular}{|c|c|c|}
\hline Variable dep: Riesgo desertar & Coeficientes & $\begin{array}{l}\text { Errores estándar } \\
\quad \text { Robustos }\end{array}$ \\
\hline Género (Hombres = 1) & $0,278^{* * *}$ & $(0.0530)$ \\
\hline Trabajaba pruebas Saber $11(\mathrm{Si}=1)$ & $0,352^{* * *}$ & $(0.0834)$ \\
\hline Modalidad $($ Distancia $=1)$ & $0,208^{*}$ & $(0.117)$ \\
\hline \multicolumn{3}{|l|}{ Nivel educativo de la madre } \\
\hline Secundaria & $-0,00184$ & $(0.0617)$ \\
\hline Técnico / Tecnológico & $-0,138$ & (0.106) \\
\hline Universitario & $-0,141$ & (0.102) \\
\hline \multicolumn{3}{|l|}{ Ingresos familiares } \\
\hline 1 a 2 salarios mínimos & $-0,019$ & $(0.0595)$ \\
\hline 2 a 3 salarios mínimos & $-0,109$ & $(0.0909)$ \\
\hline 3 a 5 salarios mínimos & 0,0771 & $(0.147)$ \\
\hline 5 a 7 salarios mínimos & 0,0513 & $(0.279)$ \\
\hline 7 a 10 salarios mínimos & $-0,678^{*}$ & $(0.403)$ \\
\hline más de 10 salarios mínimos & 0,925 & $(1.024)$ \\
\hline \multicolumn{3}{|l|}{ Clasificación pruebas Saber 11} \\
\hline Medio & $-0,247^{* * *}$ & $(0.0641)$ \\
\hline Alto & $-0,210^{* *}$ & $(0.100)$ \\
\hline \multicolumn{3}{|l|}{ Edad momento prueba Saber 11} \\
\hline 15 años o menos & $-0,15$ & (0.129) \\
\hline 16 a 20 años & $-0,160 * *$ & $(0.0680)$ \\
\hline 21 a 25 años & $-0,341^{* * *}$ & $(0.110)$ \\
\hline \multicolumn{3}{|l|}{ Apoyos académicos recibidos } \\
\hline 1 apoyo recibido & $-0,0861$ & $(0.125)$ \\
\hline 2 apoyos recibidos & $-0,999^{*}$ & (0.594) \\
\hline 3 apoyos recibidos & $-16,81$ & $(3,834)$ \\
\hline más de 4 apoyos recibidos & $0,375^{* *}$ & $(0.157)$ \\
\hline \multicolumn{3}{|l|}{ Apoyos financieros recibidos } \\
\hline 1 apoyo recibido & $-1,040^{* * *}$ & (0.178) \\
\hline 2 apoyos recibidos & $-0,942^{* * *}$ & $(0.180)$ \\
\hline 3 apoyos recibidos & $-1,502^{* * *}$ & $(0.154)$ \\
\hline más de 4 apoyos recibidos & - & \\
\hline \multicolumn{3}{|l|}{ Estrato socioeconómico } \\
\hline Estrato 2 & 0,0481 & $(0.0624)$ \\
\hline Estrato 3 & 0,0819 & $(0.111)$ \\
\hline Estrato 4 & 0,258 & $(0.281)$ \\
\hline Estrato 5 & $-0,444$ & (0.541) \\
\hline Estrato 6 & 0,386 & $(12,872)$ \\
\hline
\end{tabular}


Tabla 5.

Modelo de duración Universidad de Cartagena, Periodo 2012-I a 2016-I

\begin{tabular}{|c|c|c|}
\hline Variable dep: Riesgo desertar & Coeficientes & $\begin{array}{l}\text { Errores estándar } \\
\quad \text { Robustos }\end{array}$ \\
\hline \multicolumn{3}{|l|}{ Tasa de repitencia } \\
\hline 0 a $10 \%$ & $-21,27$ & (833.9) \\
\hline 10 a $20 \%$ & $-1,980^{* * *}$ & (0.137) \\
\hline 20 a $30 \%$ & $1,804^{* * *}$ & (0.179) \\
\hline 30 a $40 \%$ & $-0,941^{* * *}$ & $(0.180)$ \\
\hline 40 a $50 \%$ & $1,314^{* * *}$ & $(0.138)$ \\
\hline \multicolumn{3}{|l|}{ Tamaño de la familia } \\
\hline 1 a 3 integrantes & 0,207 & $(0.182)$ \\
\hline 4 a 6 integrantes & 0,0633 & (0.178) \\
\hline 7 a 9 integrantes & 0,132 & $(0.188)$ \\
\hline Constante & $-3,153^{* * *}$ & $(0.271)$ \\
\hline In_p & $0.460^{* * *}$ & $(0.0196)$ \\
\hline Observaciones & 3,180 & \\
\hline
\end{tabular}

Errores estándar robustos en paréntesis

${ }^{* * *} \mathrm{p}<0.01,{ }^{* *} \mathrm{p}<0.05,{ }^{*} \mathrm{p}<0.1$

Fuente: Elaboración de los autores con base en SPADIES 2.8

En cuanto al riesgo de desertar de acuerdo a la metodología de estudio presencial y a distancia. La Tabla 6, contiene los resultados para los programas presenciales agrupados en áreas de conocimiento. Nuevamente los hombres presentan un riesgo mayor de desertar respecto a las mujeres en un 18,2\% con una significancia estadística de un $5 \%$, aquellos que trabajaban al momento de presentar las pruebas Saber 11 presentan un 25,4\% de riesgo a desertar superior a los que no trabajaban en ese momento (Aleans, 2012).

En relación al nivel educativo de la madre, aquellos estudiantes en los que su madre alcanzó un nivel educativo de secundaria, técnico/tecnológico y universitario muestran un riesgo menor de desertar en comparación de aquellos donde su mamá solo alcanzó la primaria en $22 \%, 42,2 \%$ y $55,6 \%$, en ese mismo orden. Respecto a la clasificación de las pruebas Saber 11, aquellos que obtuvieron una clasificación media y alto presentan un riesgo menor de desertar en un 12\%, en comparación con los que se ubican en nivel bajo, estos resultados son consistentes con los hallados en Aleans (2012) y Escorcia (2013).

En cuanto a los apoyos académicos y financieros, se corrobora una vez más que recibir uno o dos apoyos reduce significativamente el riesgo de desertar en lugar de no recibir ningún tipo de ayuda. En ese mismo sentido, recibir demasiados apoyos en comparación de no recibir ninguno de ellos, representa un riesgo mayor de desertar (Castillo, 2009)

Analizando por área de conocimiento, se tomó como categoría de comparación las ciencias naturales y exactas (matemáticas, química y biología) dado que se 
presentaban altas tasas de deserción. Al compararse con las demás disciplinas, se halló que pertenecer a la facultad de ingeniera genera un riesgo mayor (7\%) que en ciencias exactas, pero en ciencias económicas, humanas, de la salud, sociales y derecho, muestran un riesgo menor en $64 \%, 17 \%, 67 \%, 45,7 \%$ y $21,8 \%$, respectivamente.

Tabla 6.

Modelos de duración programas Presenciales

\begin{tabular}{|c|c|c|}
\hline Variable dep: Riesgo desertar & Coeficientes & $\begin{array}{l}\text { Errores estándar } \\
\quad \text { Robustos }\end{array}$ \\
\hline Género $($ Hombres = 1) & $0,182^{* *}$ & $(0.012)$ \\
\hline Trabajaba pruebas Saber $11(\mathrm{Si}=1)$ & $0,254^{*}$ & $(0.051)$ \\
\hline \multicolumn{3}{|l|}{ Nivel educativo de la madre } \\
\hline Secundaria & $-0,22^{*}$ & $(0.218)$ \\
\hline Técnico / Tecnológico & $-0,422^{* *}$ & $(0.273)$ \\
\hline Universitario & $-0,556^{*}$ & $(0.264)$ \\
\hline \multicolumn{3}{|l|}{ Ingresos familiares } \\
\hline 1 a 2 salarios mínimos & $0,174^{* *}$ & $(0.167)$ \\
\hline 2 a 3 salarios mínimos & 0,303 & $(0.002)$ \\
\hline 3 a 5 salarios mínimos & 0,659 & $(0.313)$ \\
\hline 5 a 7 salarios mínimos & 0,325 & $(0.497)$ \\
\hline 7 a 10 salarios mínimos & 0,6172 & $(0.620)$ \\
\hline más de 10 salarios mínimos & $-13,63$ & $(4,340)$ \\
\hline \multicolumn{3}{|l|}{ Clasificación pruebas Saber 11} \\
\hline Medio & $-0,12^{* *}$ & $(0.169)$ \\
\hline Alto & $-0,119^{*}$ & $(0.171)$ \\
\hline \multicolumn{3}{|l|}{ Edad momento prueba Saber 11} \\
\hline 15 años o menos & $0,736^{*}$ & $(0.426)$ \\
\hline 16 a 20 años & 0,0613 & $(0.351)$ \\
\hline 21 a 25 años & 0,44 & (0.383) \\
\hline \multicolumn{3}{|l|}{ Apoyos académicos recibidos } \\
\hline 1 apoyo recibido & $-0,0324^{* * *}$ & $(0.044)$ \\
\hline 2 apoyos recibidos & $-1,288^{* *}$ & $(0.616)$ \\
\hline 3 apoyos recibidos & $-14,11$ & $(2,565)$ \\
\hline más de 4 apoyos recibidos & $0,255^{*}$ & $(0,162)$ \\
\hline \multicolumn{3}{|l|}{ Apoyos financieros recibidos } \\
\hline 1 apoyo recibido & $-1,830^{*}$ & $(1.084)$ \\
\hline 2 apoyos recibidos & $-2,099^{*}$ & (1.111) \\
\hline 3 apoyos recibidos & $3,273^{* * *}$ & (0.513) \\
\hline más de 4 apoyos recibidos & $3,348^{* * *}$ & (0.493) \\
\hline \multicolumn{3}{|l|}{ Estrato socioeconómico } \\
\hline Estrato 2 & $-0,199$ & $(0.150)$ \\
\hline Estrato 3 & $-0,149$ & $(0.219)$ \\
\hline Estrato 4 & $-0,417$ & $(0.422)$ \\
\hline Estrato 5 & 0,28 & $(0.613)$ \\
\hline Estrato 6 & 0,00126 & $(8,697)$ \\
\hline
\end{tabular}


Tabla 6.

Modelos de duración programas Presenciales

\begin{tabular}{l|lc}
\hline Variable dep: Riesgo desertar & Coeficientes & $\begin{array}{c}\text { Errores estándar } \\
\text { Robustos }\end{array}$ \\
\hline Tasa de repitencia & & \\
O a 10\% & $-0,79$ & $(754.3)$ \\
10 a $20 \%$ & 0,358 & $(0.479)$ \\
20 a 30\% & $0,677^{* *}$ & $(1.129)$ \\
30 a 40\% & $-1,174$ & $(1.106)$ \\
40 a 50\% & $0,498^{* * *}$ & $(0.648)$ \\
Áreas de Conocimiento-Presencial & & \\
Ingenierías & & \\
Ciencias Económicas & $0,0688^{*}$ & $(0.198)$ \\
Ciencias Humanas & $-0,644^{* * *}$ & $(0.185)$ \\
Ciencias de la Salud & $-0,171^{*}$ & $(0.192)$ \\
Ciencias Sociales & $-0,671^{* * *}$ & $(0.234)$ \\
Derecho & $-0,457$ & $(0.360)$ \\
& $-0,218^{* *}$ & $(0.358)$ \\
Constante & & $(0.795)$ \\
In_p & $-7,374^{* * *}$ & $(0.0444)$ \\
Observaciones & $0,642^{* * *}$ & \\
\hline
\end{tabular}

Errores estándar robustos en paréntesis

${ }^{* * *} \mathrm{p}<0.01,{ }^{* *} \mathrm{p}<0.05, \quad{ }^{*} \mathrm{p}<0.1$

Fuente: Elaboración de los autores con base en SPADIES 2.8

Abordando los resultados para los programas a distancia, la Tabla 7 presenta nuevamente que los hombres tienen un riesgo mayor que las mujeres en $23,1 \%$, y los que trabajaban al momento de presentar las pruebas Saber 11 un riesgo 38\% mayor que aquellos que no trabajaban.

En cuanto al nivel educativo de la madre, los ingresos familiares y el estrato socioeconómico, los parámetros no fueron significativos por lo que no contribuyen de manera amplia a la explicación del riesgo a desertar, y esto es explicado en que entre los estudiantes de distancia poseen características muy similares en estos aspectos. No obstante, en la clasificación de las pruebas Saber 11 aquellos que obtuvieron una calificación media y alta presentan en promedio un poco más de $20 \%$ menos de riesgo que aquellos tuvieron una clasificación baja.

Ahora bien, en los apoyos recibidos SPADIES no reportó mucha información sobre los académicos por lo que se asume que por el carácter de la modalidad no permitía el escenario para que se brindaran suficientes apoyos académicos. Pero en los apoyos financieros, se muestra como al recibir uno o dos disminuye de manera significativa el riesgo a desertar, asimismo recibir más de cuatro apoyos incrementa el riesgo de desertar en un $13,3 \%$.

A diferencia de los estudiantes presenciales, el estrato socioeconómico si ayuda a explicar el riesgo a desertar, encontrando que aquellos que pertenecen al estrato 
2 y 3 tienen un riesgo a desertar 15,7\% y 25,3\% mayor a desertar que aquellos que pertenecen al estrato 1 . Estos resultados son interesantes en la medida en que se esperaría un signo contrario, pero el hecho de que SPADIES no los desagregue por centro tutorial limita el análisis al ser lugares con características socioeconómicas totalmente distintas.

Respecto a la tasa de repitencia, aquellos estudiantes que tuvieron una tasa menor al $50 \%$ presentan un riesgo menor de desertar casi 2 veces mayor como el caso del rango de $0 \%$ a $10 \%$ de repitencia comparados con el rango de $50 \%$ y $60 \%$.

Tabla 7.

Modelos de duración programas a Distancia

\begin{tabular}{|c|c|c|}
\hline Variable dep: Riesgo desertar & Coeficientes & $\begin{array}{c}\text { Errores estándar } \\
\text { Robustos }\end{array}$ \\
\hline Género (Hombres = 1) & $0,231^{* * *}$ & $(0.0615)$ \\
\hline Trabajaba pruebas Saber $11(\mathrm{Si}=1)$ & $0,380^{* * *}$ & $(0.0983)$ \\
\hline \multicolumn{3}{|l|}{ Nivel educativo de la madre } \\
\hline Secundaria & $-0,0731$ & $(0.0658)$ \\
\hline Técnico / Tecnológico & -0.104 & $(0.126)$ \\
\hline Universitario & -0.0680 & $(0.118)$ \\
\hline \multicolumn{3}{|l|}{ Ingresos familiares } \\
\hline 1 a 2 salarios mínimos & -0.0421 & $(0.0650)$ \\
\hline 2 a 3 salarios mínimos & -0.158 & (0.108) \\
\hline 3 a 5 salarios mínimos & 0.0686 & $(0.176)$ \\
\hline 5 a 7 salarios mínimos & 0.130 & $(0.361)$ \\
\hline 7 a 10 salarios mínimos & -0.454 & $(0.587)$ \\
\hline \multicolumn{3}{|l|}{ más de 10 salarios mínimos } \\
\hline \multicolumn{3}{|l|}{ Clasificación pruebas Saber 11} \\
\hline Medio & $-0,241^{* * *}$ & $(0.0711)$ \\
\hline Alto & $-0,225$ & $(0.153)$ \\
\hline \multicolumn{3}{|l|}{ Edad momento prueba Saber 11} \\
\hline 15 años o menos & -0216 & $(0.143)$ \\
\hline 16 a 20 años & $-0.171^{* * *}$ & $(0.0818)$ \\
\hline 21 a 25 años & $-0.422^{* * *}$ & $(0.120)$ \\
\hline \multicolumn{3}{|l|}{ Apoyos académicos recibidos } \\
\hline 1 apoyo recibido & -13.04 & $(1,799)$ \\
\hline \multicolumn{3}{|l|}{ Apoyos financieros recibidos } \\
\hline 1 apoyo recibido & $-1,062^{* * *}$ & $(0.194)$ \\
\hline 2 apoyos recibidos & $-0.880^{* * *}$ & $(0.187)$ \\
\hline 3 apoyos recibidos & $1.329^{* * *}$ & $(0.168)$ \\
\hline más de 4 apoyos recibidos & 0.133 & $(0.175)$ \\
\hline
\end{tabular}


Tabla 7.

Modelos de duración programas a Distancia

\begin{tabular}{lll}
\hline Variable dep: Riesgo desertar & Coeficientes & $\begin{array}{c}\text { Errores estándar } \\
\text { Robustos }\end{array}$ \\
\hline Estrato socioeconómico & & \\
Estrato 2 & $0.157^{* *}$ & $(0.0709)$ \\
Estrato 3 & $0.253^{*}$ & $(0.137)$ \\
Estrato 4 & 0.184 & $(0.463)$ \\
Estrato 5 & 0.456 & $(6.403)$ \\
Estrato 6 & & \\
Tasa de repitencia & & \\
O a 10\% & & $(448.6)$ \\
10 a 20\% & -19.84 & $(0.151)$ \\
20 a 30\% & $-2.288^{* * *}$ & $(0.191)$ \\
30 a 40\% & $1.235^{* * *}$ & $(0.193)$ \\
40 a 50\% & $-1.034^{* * *}$ & $(0.143)$ \\
Programas a Distancia & $0.805^{* * *}$ & \\
Administración de Empresas & & $(0.119)$ \\
Ingeniería de Sistemas & & $(0.106)$ \\
Administración Servicios Salud & $-0.226^{*}$ & $(0.102)$ \\
Administración Financiera & $0.146^{* *}$ & $(0.107)$ \\
Tecnologías & $0.0444^{*}$ & $(0.276)$ \\
& $-0.220^{* *}$ & \\
Constante & $0.105^{*}$ & $(0.196)$ \\
In_p & & $(0.0219)$ \\
Observaciones & & \\
\hline
\end{tabular}

Errores estándar robustos en paréntesis

${ }^{* * *} \mathrm{p}<0.01,{ }^{* *} \mathrm{p}<0.05,{ }^{*} \mathrm{p}<0.1$

Fuente: Elaboración de los autores con base en SPADIES 2.8

Finalmente, se planteó una pregunta abierta en la que los encuestados podían escribir alguna otra razón por la que desertaron. Dichas respuestas fueron agrupadas en las categorías plasmadas en la Tabla 8, encontrado que en los programas presenciales la principal razón es que manifestaron que la carrera que eligieron no era de su preferencia $(29,8 \%)$, esta respuesta tuvo el segundo mayor peso en los encuestados de distancia (19,4\%), siendo los problemas económicos la principal razón de abandono (25,7\%). Entre otras respuestas en las que ambas modalidades coinciden son: problemas personales, falta de tiempo, falta de infraestructura (laboratorios, salas de sistemas, salones, servicios públicos, etc.), poca motivación, problemas con docentes, inflexibilidad curricular, entre otras. 
Tabla 8.

Razones expresadas por los participantes por las que desertaron, 2012-I a 2016-I

\begin{tabular}{lcc}
\hline Principales razones & Presencial & Distancia \\
\hline No era de mi preferencia & $29,8 \%$ & $19,4 \%$ \\
Problemas económicos & $19,3 \%$ & $25,7 \%$ \\
Problemas personales & $10,5 \%$ & $17,4 \%$ \\
Falta de tiempo & $8,8 \%$ & $8,3 \%$ \\
Falta de infraestructura & $5,3 \%$ & $4,2 \%$ \\
Poca motivación & $5,3 \%$ & $4,9 \%$ \\
Bajo rendimiento académico & $3,5 \%$ & $3,5 \%$ \\
Problemas con docentes & $3,5 \%$ & $6,3 \%$ \\
Ambiente tenso & $1,8 \%$ & $0,0 \%$ \\
Apoyo académico & $1,8 \%$ & $2,8 \%$ \\
Embarazo & $1,8 \%$ & $2,1 \%$ \\
Inflexibilidad curricular & $1,8 \%$ & $3,5 \%$ \\
Me fui del país & $1,8 \%$ & $0,0 \%$ \\
Me iba atrasando & $1,8 \%$ & $0,0 \%$ \\
Problemas de salud & $0,0 \%$ & $1,4 \%$ \\
\hline Total & $100 \%$ & $100 \%$ \\
\hline
\end{tabular}

Fuente: Camacho M, Montalvo A, Galezo P. Factores que determinan la deserción estudiantil en estudiantes de pregrado. Universidad de Cartagena 2012-1- 2016-1

\section{CONCLUSIONES}

Si bien los resultados evidenciaron una disminución considerable en las tasas de deserción de la Universidad de Cartagena a lo largo del tiempo, esta va en sincronía con el comportamiento de la tasa promedio nacional. Esto no implica que se deben descuidar los mecanismos de prevención para la retención estudiantil y poder fortalecerlos para intentar erradicar esta problemática.

De acuerdo a los resultados arrojados por el componente descriptivo y correlacional del estudio, es una prioridad de la Vicerrectoría de Bienestar Institucional el atender a la población masculina y a la población en general de los programas a distancia. Además, la Universidad debe identificar e intervenir a la población que ingresa con clasificación baja en las pruebas Saber 11, dado que se comprobó correlación entre estos estudiantes y las altas tasas de deserción.

En ese mismo sentido, de acuerdo a los resultados obtenidos que en la Universidad de Cartagena también se presenta una alta deserción temprana más que tardía. Por tanto, los esfuerzos se deben orientar a los primeros semestres de formación, sin descuidar los demás periodos.

Por otra parte, aunque la Universidad tiene limitaciones frente a aspectos como el nivel educativo de la madre, los ingresos familiares, y el estrato, al no poder controlarlas 
de manera directa, por lo que debe diseñar y planear desde las diferentes áreas y departamentos de la Universidad estrategias orientadas a disminuir el efecto de estas variables sobre el riesgo a desertar.

No obstante, la institución si tiene otras formas de poder mitigar el efecto de la decisión de abandonar los estudios respecto a variables como la tasa de repitencia, apoyos académicos y financieros, la motivación, flexibilidad curricular, la adecuada dotación en infraestructura, el componente vocacional, las relaciones interpersonales y apoyos psicosociales.

Por último, la sección encargada del Bienestar Estudiantil en la Vicerrectoría de Bienestar Institucional además de establecer esfuerzos alrededor de esta temática, debe hacer un continuo seguimiento de la información suministrada a SPADIES para evitar enviar información incompleta o con errores que impiden la precisión de los análisis respectivos.

\section{REFERENCIAS BIBLIOGRÁFICAS}

Aleans, K. (2012). Determinantes de la deserción estudiantil universitaria por niveles de formación en Instituciones de Educación Superior de la ciudad de Medellín. Universidad EAFIT Repositorio Institucional. 212: 48 Recuperado de: https://repository.eafit.edu.co/ handle/10784/610

Viloria, J, Peralta, A, Escorcia, R. (2013). Caracterización de los estudiantes de la Universidad Popular del Cesar de acuerdo a las variables socioeconómicas, psicosociales y geográficas. Editorial: UNICESAR. Recuperado de: http://editorial.unicesar.edu.co/index. $\mathrm{php} /$ libros/por-facultades/ciencias-basicas-y-de-educacion/52-caracterizacion-de-losestudiantes-de-la-universidad-popular-del-cesar-de-acuerdo-a-las-variablessocioeconomicas-sicosociales-y-geograficas

Castillo, M, Sánchez A, Venegas, F. (2009). La modelación económica. Una interpretación de la simulación dinámica de sistemas. $1^{\text {a }}$ ed. México: Ediciones y Gráficos Eón, S.A. Recuperado de: https://core.ac.uk/download/pdf/83079903.pdf

Giovagnoli, P. (2002). Determinantes de la Deserción y Graduación universitaria: Una aplicación utilizando modelos de duración. Universitaria. Universidad Nacional de La Plata (Argentina). Recuperado de: http://www.alfaguia.org/alfaguia/files/ 1318958623Determinantes\%20de\%20la\%20desercion\%20y\%20graduacion $\% 20$ universitaria\%20una\%20aplicacion $\% 20$ utilizando\%20 modelos\%20de\%20 duracion.pdf

Lopera, C. (2008). Determinantes de la deserción universitaria en la Facultad de Economía Universidad del Rosario. Borradores de Investigación. Universidad del Rosario. Recuperado de: http://repository.urosario.edu.co/bitstream/handle/10336/3740/ Borrador_Economia_95\%5B1\%5D.pdf?sequence=8\&isAllowed=y

Páramo, G, Correa, C. (1999). Deserción estudiantil universitaria. Conceptualización. Revista Universidad EAFIT, 35 (114): 65-78. Recuperado de: http://publicaciones.eafit.edu.co/index. $\mathrm{php} /$ revista-universidad-eafit/article/view/1075

Ministerio de Educación Nacional (2015). Guía para la implementación de Educación Superior del modelo de gestión de permanencia y graduación estudiantil en instituciones de Educación Superior. Recuperado de: http://www.mineducacion.gov.co/1759/w3-article-356272.html 
Ministerio de Educación Nacional. (2015). Todos por un nuevo país PAZ EQUIDAD Educación. Estrategias para la permanencia en Educación Superior: Experiencias Significativas. QUALIFICAR. Recuperado de: http://www.colombiaaprende.edu.co/html/micrositios/ 1752/articles-350844_pdf.pdf

Ministerio de Educación Nacional (2009). Revolución educativa Colombia aprende. Deserción estudiantil en la Educación Superior Colombiana. Metodología de seguimiento, diagnóstico y elementos para su prevención. Imprenta Nacional de Colombia. Recuperado de: http://www.mineducacion.gov.co/sistemasdeinformacion/1735/articles-254702_ libro_desercion.pdf

Ministerio de Salud (1993). Resolución 008430 de 1993 por el cual se definen se establecen las normas científicas, técnicas y administrativas para la investigación en salud. Bogotá: Ministerio de Salud. Recuperado de: https://www.invima.gov.co/images/pdf/ tecnovigilancia/buenas_practicas/normatividad/Resolucion_8430_de_1993.pdf

Universidad de Cartagena, (2012). Encuesta deserción estudiantil, Vicerrectoría de Bienestar Institucional: 92-93.

Universidad de Cartagena (2012). Diagnostico institucional sobre factores determinantes de la deserción estudiantil en los programas de pregrado en la Universidad de Cartagena. Sistema Integrado de Retención Estudiantil (SIRE). Documento de trabajo. 